\title{
Accretion discs models with the $\beta$-viscosity prescription derived from laboratory experiments
}

\author{
J.-M. Huré1,2, D. Richard ${ }^{2,3}$, and J.-P. Zahn ${ }^{3}$ \\ 1 DAEC et UMR 8631 du CNRS, Observatoire de Paris, Place Jules Janssen, 92195 Meudon Cedex, France \\ 2 Université Paris 7 Denis Diderot, 2 place Jussieu, 75251 Paris Cedex 05, France \\ 3 DASGAL et UMR 8633 du CNRS, Observatoire de Paris, Place Jules Janssen, 92195 Meudon Cedex, France \\ Received 7 September 2000 / Accepted 20 November 2000
}

\begin{abstract}
We examine under which conditions one may apply, to steady state Keplerian accretion discs, the $\beta$-viscosity prescription which has been derived from rotating shear flow experiments $\left(\nu=\beta \Omega R^{2}\right.$, where $\Omega$ is the angular velocity at radius $R$ and $\beta$ is a constant of order $10^{-5}$; Richard \& Zahn 1999). Using a vertically averaged model, we show that this law may be suitable for all three families of known systems: in young stellar objects, evolved binary stars and Active Galactic Nuclei discs (except in their outer gas pressure dominated regions where turbulence becomes hypersonic). According to the standard criterion for viscous stability, $\beta$-discs are always stable throughout. Using realistic opacities and equation of state, we demonstrate that these discs are thermally unstable in the temperature domain where hydrogen recombines, when they are optically thick, and this could lead to limit cycle behavior. Radiation pressure dominated regions are thermally stable, in contrast with $\alpha$-discs. This results in a fully stable solution for the innermost parts of AGN discs.
\end{abstract}

Key words. accretion, accretion discs - instabilities - hydrodynamics - galaxies: active - galaxies: nuclei

\section{Introduction}

What causes the transport of matter and angular momentum in accretion discs? This question is the subject of a longstanding debate, but most of those working in this field tend now to agree that it has been answered, since even a very weak magnetic field leads to a linear instability (Chandrasekhar 1961) which, in its fully developed regime, is able to provide the necessary stresses (Balbus \& Hawley 1991; Hawley \& Balbus 1991). However, it is unlikely that this mechanism operates in cold neutral discs (e.g. Gammie \& Menou 1998). Besides, the issue which has not been settled yet is whether this magnetic instability is the only instability responsible for the "anomalous viscosity", or whether a purely hydrodynamic instability, generated by shear in differentially rotating discs, may not be of comparable or even higher efficiency. Since a non-magnetic Keplerian disc is linearly stable, this hydrodynamic instability needs a finite amplitude perturbation to be triggered and laboratory experiments indicate that it appears only above Reynolds numbers of the order of $10^{4}$. Such conditions are still out of reach of the most ambitious numerical simulations; therefore, these cannot be used yet to prove or to disprove the occurrence of shear instability.

Send offprint requests to: J.-M. Huré, e-mail: Jean-Marc.Hure@obspm.fr
It is worth noting that the widely used $\alpha$-prescription for turbulent viscosity (Shakura \& Sunyaev 1973) does not invoke any particular mechanism at the origin of turbulence: it is a simple parameterization which is tailored to yield turbulent velocities that remain subsonic (or subalfvénic) for $\alpha<1$. It is often asserted that the main success of the $\alpha$-prescription is the prediction of recurrent outbursts of dwarf novae (e.g. Cannizzo 1993). However, if such eruptive phenomena are indeed of thermal origin, as is commonly thought, it is likely that many ad-hoc viscosities, not only the $\alpha$-prescription, could work as well.

For these multiple reasons it makes sense to investigate the basic properties of accretion discs built with the alternate $\beta$-prescription, which is observed in laboratory rotating shear flows, as explained below. This is our intention in this paper. We first present in Sect. 2 some properties of $\beta$-viscosity, and examine in Sect. 3 to which regimes it may be applied, allowing for subsonic turbulent velocities in geometrically thin, radiatively cooled accretion discs. In Sect. 4 we study the viscous and thermal stabilities of $\beta$-discs, using standard criteria, and draw our conclusions in the last section. The Appendix contains the vertically averaged equations for $\beta$-discs and the derivation of the criterion of thermal instability.

\section{Comparing the $\alpha$ and $\beta$-viscosities}

The laboratory experiment which is the most pertinent to studies of hydrodynamical instabilities in differentially 
rotating flows is the Couette-Taylor experiment: a fluid is sheared between two cylinders rotating at different speeds. Only a few experiments have been run in the case where the angular momentum increases outwards, as in a Keplerian disc, but from the torque measurements that are available, it appears that the turbulent viscosity scales as

$\nu_{\mathrm{t}} \propto R^{3}\left|\frac{\mathrm{d} \Omega}{\mathrm{d} R}\right|$,

where $R$ is the distance from the rotation axis and $\Omega$ is the angular velocity (Richard \& Zahn 1999). In a Keplerian disc, this relation is equivalent to

$\nu_{\beta}=\beta \Omega R^{2}$,

which we shall call the $\beta$-prescription.

This prescription was originally proposed by LyndenBell \& Pringle (1974) and some aspects of it have been discussed in later papers (De Freitas Pacheco \& Steiner 1976; Thompson et al. 1977; Lin \& Papaloizou 1980; Williams 1980; Hubeny 1990). It has been recently revived by Biermann \& Duschl (1998; see also Duschl et al. 2000 ) on the grounds that the only relevant scales in a Keplerian disc are the angular velocity and the radius, since they contain all the information about the rotation and the curvature of the flow. These authors make the sensible choice of equating the parameter $\beta$ with the inverse of the critical Reynolds number $\mathcal{R} e$, which they assume to be the same as in plane-parallel shear flows (i.e. $\mathcal{R} e \approx 10^{3}$ and therefore $\beta \approx 10^{-3}$ ).

However, in the following we shall use a smaller value for this $\beta$-parameter, which is derived from the CouetteTaylor experiment. The only experimental data available, in the case where angular momentum increases outwards, are those obtained by Wendt (1933) and Taylor (1936), in which the inner cylinder is at rest; from these one derives $\beta \approx 10^{-5}$. This value is compatible with the critical gradient Reynolds number which characterizes these experiments (see Richard \& Zahn 1999):

$\mathcal{R} e_{\mathrm{c}}^{*}=\frac{R^{3}}{\nu}\left|\frac{\mathrm{d} \Omega}{\mathrm{d} R}\right| \approx 610^{5}$,

where $\nu$ is the molecular viscosity of the fluid. Clearly, new experiments are required to measure this parameter in regimes which resemble more the Keplerian flow; in the meanwhile, until new results are obtained, we shall adopt the value quoted above.

One important property of this $\beta$-viscosity is that it depends only on the radius in a Keplerian disc, and does not involve local physical conditions. On the contrary, the standard $\alpha$-prescription (Shakura \& Sunyaev 1973) depends on the local values of the pressure scale height $H$ and sound velocity $c_{\mathrm{S}}$ (taken in a broad sense - it is the Alfvén velocity if magnetic pressure dominates):

$\nu_{\alpha}=\alpha c_{\mathrm{s}} H$.

To emphasize the difference between the present prescription and the standard law, we use the relation $c_{\mathrm{s}}=\Omega H$ which expresses the hydrostatic equilibrium in a Keplerian vertically averaged disc, and rewrite Eq. (4) as

$\nu_{\alpha}=\alpha \Omega R^{2}\left(\frac{H}{R}\right)_{\alpha}^{2}=\nu_{\beta} \times \frac{\alpha}{\beta} \times\left(\frac{H}{R}\right)_{\alpha}^{2}$.

We see that the ratio $\nu_{\alpha} / \nu_{\beta}$ depends on the aspect ratio of the $\alpha$-disc. In general, the quantity $(H / R)_{\alpha}$ is weakly sensitive to both $R$ and $\alpha$, but it depends strongly on the central mass and the accretion rate, and is typically in the range 0.001-0.1 (e.g. Huré 1998).

The $\alpha$-prescription has been designed such that the Mach number is smaller than unity for $\alpha \leq 1$. In contrast, subsonic turbulence is not guaranteed with the $\beta$ prescription. This restriction on subsonic turbulent velocities is dictated by the fact that supersonic turbulence would be highly dissipative and therefore difficult to sustain. Moreover, the turbulent viscosity used here has been measured in a liquid, and its application to compressible fluids can only be justified in the subsonic range. Therefore one has to check whether the $\beta$-prescription predicts subsonic turbulence in a given disc. By making the reasonable assumption that the vorticity $v_{\mathrm{t}} / \ell_{\mathrm{t}}$ of the turbulent eddies is of order $\Omega\left(v_{\mathrm{t}}\right.$ and $\ell_{\mathrm{t}}$ denoting respectively the typical velocity and length scales of turbulence), and setting $\nu_{\beta}=v_{\mathrm{t}} \ell_{\mathrm{t}}$, the Mach number in a $\beta$-disc is given by

$\mathcal{M}_{\mathrm{t}}=\frac{v_{\mathrm{t}}}{c_{\mathrm{s}}} \simeq \sqrt{\beta} \frac{\Omega R}{c_{\mathrm{s}}}=\sqrt{\beta}\left(\frac{H}{R}\right)_{\beta}^{-1}$

where $(H / R)_{\beta}$ is the aspect ratio of the $\beta$-disc. We note that $\mathcal{M}_{\mathrm{t}}<1$ provided $H / R>\sqrt{\beta}$, which is not a too severe requirement with our low value of $\beta$ (the situation would be more problematic with $\beta=10^{-3}$, conflicting with the Keplerian assumption).

\section{Application range of the $\beta$-viscosity}

Let us now examine under which conditions the $\beta$ prescription allows for subsonic turbulence in geometrically thin accretion discs in active galactic nuclei (AGN), evolved binary systems (EBS) and young stellar objects (YSO). For this first exploration, we will be content with the usual one-dimensional steady state approach to compute the sound speed and the aspect ratio, the vertical structure being reduced to vertical averages. The reader can find the relevant equation set in Appendix A.

In a stationary Keplerian disc characterized by a steady accretion rate $\dot{M}$, the surface density $\Sigma$ is determined by the conservation of mass and momentum:

$\dot{M}=3 \pi \nu \Sigma$,

ignoring a correcting factor which is negligible sufficiently far from the center (see Frank et al. 1992). Applying the $\beta$-prescription (2), we find

$$
\begin{aligned}
& \Sigma \simeq 2.4310^{5} \beta_{-5}^{-1} f_{\text {edd }} x^{-1 / 2} \quad \mathrm{~g} \mathrm{~cm}^{-2} \\
& \simeq 92 \beta_{-5}^{-1} \dot{m}_{16} M_{0}^{-1 / 2} r_{10}^{-1 / 2} \mathrm{~g} \mathrm{~cm}^{-2} \\
& \simeq 70 \beta_{-5}^{-1} \dot{M}_{-8} M_{0}^{-1 / 2} r_{\mathrm{AU}}^{-1 / 2} \mathrm{~g} \mathrm{~cm}^{-2}
\end{aligned}
$$


where $f_{\text {edd }} \simeq 4.5 \quad \dot{M}_{0} \quad M_{8}^{-1}$ is the Eddington factor (around 0.1 typically), $M=M_{8} 10^{8} M_{\odot}=M_{0} M_{\odot}$, $\dot{M}=\dot{M}_{0} M_{\odot}=\dot{m}_{16} 10^{16} \mathrm{~g} / \mathrm{s}=\dot{M}_{-8} 10^{-8} M_{\odot} / \mathrm{yr}$, $R=x R_{\mathrm{S}}=R_{10} 10^{10} \mathrm{~cm}=r_{\mathrm{AU}}$ AU $\left(R_{\mathrm{S}}=2 G M / c^{2}\right.$ being the Schwarzschild radius of the black hole), and $\beta_{-5}=\beta 10^{5}$. These dimensionless variables are well suited to describe the three families of discs quoted above. Note that Eq. (8) is quite general and does not depend on the vertical structure.

Thermal equilibrium between radiative cooling and viscous heating provides another equation. Assuming that the disc is optically thick, we have (Frank et al. 1992):

$\frac{16 \sigma T^{4}}{3 \kappa_{\mathrm{R}} \Sigma}=\frac{3 \Omega^{2} \dot{M}}{8 \pi}$

where $\sigma$ is the Stefan constant and $\kappa_{\mathrm{R}}$ the Rosseland mean opacity.

\subsection{Regimes compatible with subsonic turbulence}

In gas pressure supported discs, the hydrostatic equation yields directly the mid-plane temperature $T$ :

$\frac{P_{\mathrm{g}}}{\rho}=\Omega^{2} H^{2}=\frac{k T}{\mu \mathrm{m}_{\mathrm{H}}}$,

with the usual notations for the gas pressure $P_{\mathrm{g}}$, the density $\rho$, the Boltzmann constant $k$, the molecular weight $\mu$ and the mass of the hydrogen atom $m_{\mathrm{H}}$. It then follows from Eqs. (6), (8), (9) and (10) that the turbulent Mach number in those discs is

$$
\begin{aligned}
\mathcal{M}_{\mathrm{t}} & \simeq 3.1 \beta_{-5}^{5 / 8} \kappa_{\mathrm{R}}^{-1 / 8} \mu^{1 / 2} f_{\mathrm{edd}}^{-1 / 4} M_{8}^{1 / 8} x^{-1 / 16} \\
& \simeq 0.3 \beta_{-5}^{5 / 8} \kappa_{\mathrm{R}}^{-1 / 8} \mu^{1 / 2} \dot{m}_{16}^{-1 / 4} M_{0}^{7 / 16} r_{10}^{-1 / 16} \\
& \simeq 410^{-2} \beta_{-5}^{5 / 8} \kappa_{\mathrm{R}}^{-1 / 8} \mu^{1 / 2} \dot{M}_{-8}^{-1 / 4} M_{0}^{7 / 16} r_{\mathrm{AU}}^{-1 / 16} .
\end{aligned}
$$

Note the relatively strong dependence of the Mach number on the viscosity parameter and its weak dependence on the opacity and specially the radius.

We conclude that the $\beta$-prescription is certainly applicable to gas pressure dominated accretion discs around forming stars, white dwarfs, neutrons stars and stellar black holes, but not to such discs around supermassive black holes, where this prescription would imply supersonic turbulence.

The innermost part of accretion discs may be dominated by radiation pressure if they reach a sufficiently high temperature; this occurs for instance in standard discs surrounding black holes or compact objects. In such a case, the hydrostatic equation is written

$\frac{P_{\mathrm{r}}}{\rho}=\Omega^{2} H^{2}=\frac{8 \sigma}{3 c} \frac{T^{4} H}{\Sigma}$,

and therefore, according to (9), $H$ depends only on the accretion rate, and not on the viscosity prescription:

$H=\frac{3 \kappa_{\mathrm{R}}}{16 \pi c} \dot{M}$.

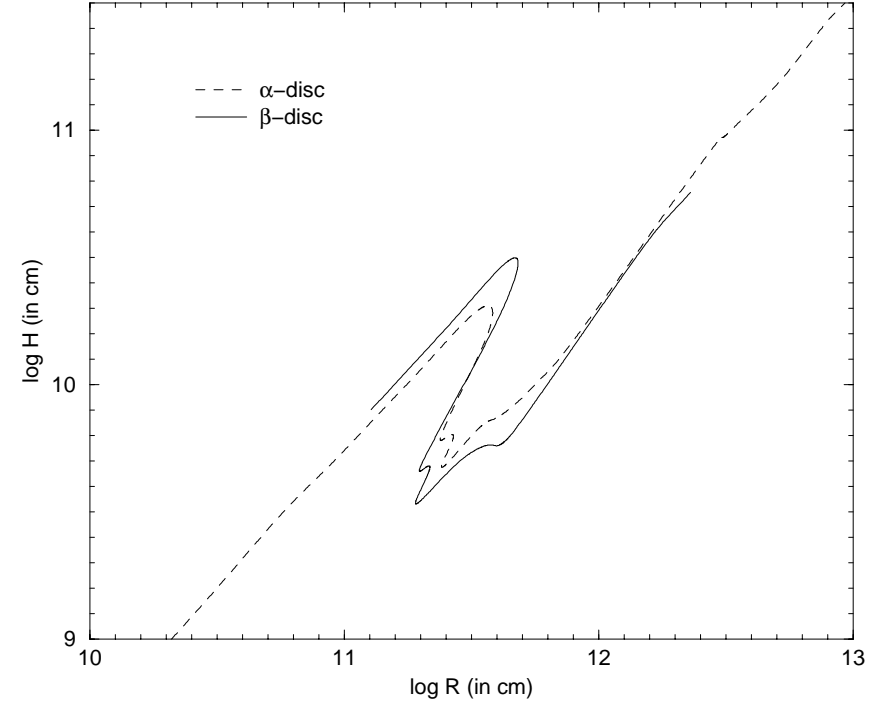

Fig. 1. Disc thickness versus radius for a $\beta$-disc with $\beta=10^{-5}$ (solid line) and for a standard disc with $\alpha=0.01$ (dashed line), for $=1 M_{\odot}$ and $\dot{M}=10^{-8} M_{\odot} /$ yr

Thus the Mach number is given by

$$
\begin{aligned}
\mathcal{M}_{\mathrm{t}} & \simeq 310^{-3} \beta_{-5}^{1 / 2} \kappa_{\mathrm{R}}^{-1} f_{\mathrm{edd}}^{-1} x \\
& \simeq 510^{-2} \beta_{-5}^{1 / 2} \kappa_{\mathrm{R}}^{-1} \dot{m}_{16}^{-1} M_{0} x
\end{aligned}
$$

meaning that turbulence is expected to be subsonic in the inner part of radiation pressure dominated discs.

\subsection{Thin Keplerian discs assumption}

In this paper, we restrict ourselves to Keplerian discs, which are necessarily geometrically thin, i.e. $H / R \ll 1$. Eq. (6) shows that the turbulent Mach number increases as the aspect ratio decreases; therefore the disc cannot be thinner than $\sqrt{\beta} \times R$. One easily finds from Eq. (11) that

$$
\begin{aligned}
\left(\frac{H}{R}\right)_{\beta} & \simeq 1.510^{-3} \kappa_{\mathrm{R}}^{1 / 8} \beta_{-5}^{-1 / 8} \mu^{-1 / 2} m_{0}^{-7 / 16} \dot{m}_{16}^{1 / 4} r_{10}^{1 / 16} \\
& \simeq 0.08 \kappa_{\mathrm{R}}^{1 / 8} \beta_{-5}^{-1 / 8} \mu^{-1 / 2} m_{0}^{-7 / 16} \dot{m}_{-7}^{1 / 4} r_{\mathrm{AU}}^{1 / 16}
\end{aligned}
$$

within the gas pressure dominated regions. It is interesting again to make the comparison with standard discs. Using power law solutions of $\alpha$-discs (Huré 1998) and Eq. (15), we obtain

$\frac{\mathrm{H}_{\beta}}{\mathrm{H}_{\alpha}} \simeq 0.9 \beta_{-5}^{1 / 8} \alpha^{1 / 10}$,

indicating that $\beta$-discs globally should have about the same shape as standard $\alpha$-discs. This is confirmed in Fig. 1, where we have plotted the disc thickness computed from vertically averaged equations with realistic opacities and equations of state, for the two viscosity laws and for $M=1 M_{\odot}$ and $\dot{M}=10^{-8} M_{\odot} /$ yr which could represent both a YSO disc and a EBS disc. In the innermost part of AGN, where the radiation pressure dominates, the disc thickness is independent of the viscosity prescription, 


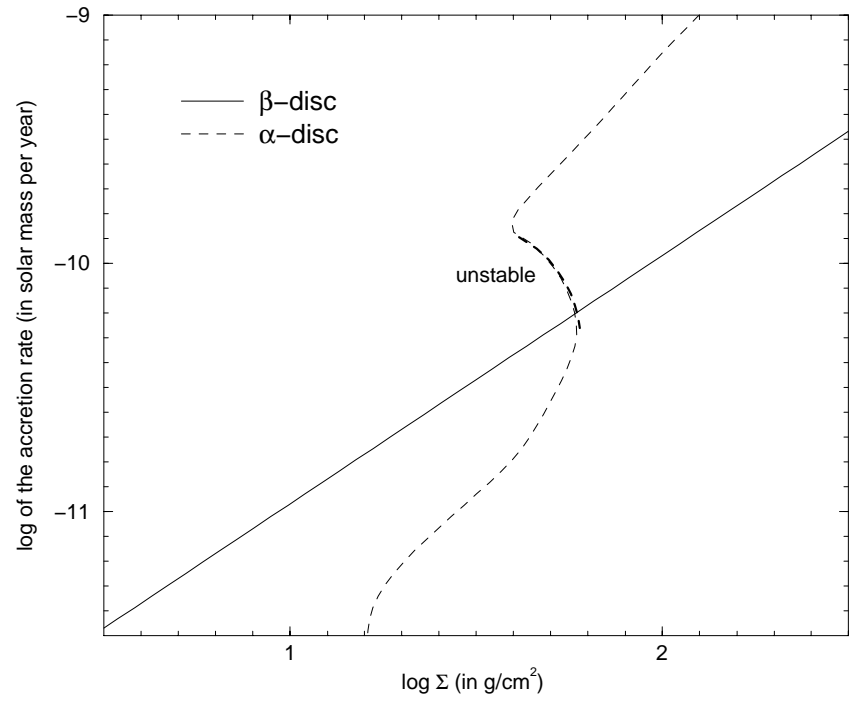

Fig. 2. Relation between the accretion rate and the surface density, from vertical structure computations, at $R=10^{10} \mathrm{~cm}$ from the center and with $M=1 M_{\odot}$, using the $\alpha$-viscosity prescription (with $\alpha=0.1$ ) and the $\beta$-prescription $\left(\beta=10^{-5}\right)$

as we have already pointed out, and therefore $\mathrm{H}_{\beta}=\mathrm{H}_{\alpha}$. We thus conclude that, despite the flaring, $\beta$-discs are expected to be thin and Keplerian, provided that the accretion rate does not reach too large values. Note that $\alpha$-discs are subject to the same constraint.

\section{Stability of $\beta$-discs}

We shall now examine two kinds of instability that an accretion disc can undergo: viscous instability which can lead to disc fragmentation and thermal instability.

\subsection{Viscous stability}

A general condition for viscous stability is (Frank et al. 1992)

$$
\left(\frac{\partial \ln \dot{M}}{\partial \ln \Sigma}\right)_{\Omega}>0 .
$$

In a standard $\alpha$-disc, the equilibrium relation $\dot{M}(\Sigma)$ takes the famous "S"-shape with the instability occuring on the intermediate branch of negative slope (Cannizzo 1993). On the contrary, according to Eqs. (2) and (7) $\beta$-discs are viscously stable everywhere, since in Eq. (17) is always fulfiled. We illustrate this important property in Fig. 2, with two typical $\dot{M}(\Sigma)$-relations obtained through vertical structure computations for a $\beta$-disc and a $\alpha$-disc (Huré 2000).

\subsection{Thermal stability}

Thermal instability occurs when the energy balance between radiative losses and viscous heating can no longer be maintained after perturbation of the equilibrium state. The condition for stability is written (Pringle 1981):

$$
\left(\frac{\partial \ln Q^{+}}{\partial \ln T}\right)_{\Sigma, \Omega}-\left(\frac{\partial \ln Q^{-}}{\partial \ln T}\right)_{\Sigma, \Omega}<0
$$

where $Q^{+}$is the heating rate (right-hand-side of Eq. (9)) and $Q^{-}$is the cooling rate (with $Q^{+}=Q^{-}$at equilibrium). The above criterion is quite general and can be applied to non standard viscosity prescriptions, provided that the vertical dynamical time scale $t_{z} \sim H / c_{\mathrm{s}}$ is smaller than the thermal time scale $t_{\mathrm{th}} \sim P H / Q^{+}$. The requirement of a subsonic turbulence discussed in the previous section automatically ensures that $t_{z} \lesssim t_{\text {th }}$ since we have the relation

$\frac{t_{\mathrm{th}}}{t_{z}} \sim \frac{1}{\beta}\left(\frac{H}{R}\right)^{2}=\frac{1}{\mathcal{M}_{\mathrm{t}}^{2}}$.

It is easy to see that $Q^{+} \propto \Omega^{3} R^{2} \Sigma$ with the $\beta$-prescription and so the thermal stability of a $\beta$-disc is only governed by properties of the cooling function. The situation is different in an $\alpha$-disc where the heating rate $Q^{+} \propto \Omega^{3} H^{2} \Sigma$ is implicitly a function of $T$ through $H$. To determine the slope of the cooling function $Q^{-}(T)$, we have used vertically averaged equations (see Appendix A) and computed the term $\left(\partial \ln Q^{-} / \partial \ln T\right)$ analytically (see Appendix B).

The resulting expression Eq. (B.7) may be used to analyze the thermal stability for very simple ideal cooling functions: electron scattering and free-free processes, as considered already by Piran (1978). We have distinguished 4 cases: optically thick, optically thin, gas pressure dominated and radiation pressure dominated. This can easily be handled by suitable settings in Eq. (B.7) (see Eqs. (B.8) and (B.11)). The results are summarized in Table 1. The remarkable point is that $\beta$-discs are always thermally stable for these ideal cooling mechanisms, which is in complete agreement with the conclusions drawn by Piran (1978). Note that the optically thin gas pressure dominated regime with free-free emission is marginally stable whereas it is unstable with the standard viscosity law, as noticed by Pringle et al. (1973).

The situation is quite different when using realistic Rosseland and Planck opacities as well as an accurate equation of state. A typical result is displayed in Fig. 3, where we have considered a disc surrounding a one solar mass central object and $\dot{M}=10^{-8} M_{\odot} /$ yr. We find that the disc is thermally unstable when the midplane temperature is in the range 5000-9000 K (zone 2 in the figure), due to the steep rise in the opacity. Like in a standard disc, this unstable regime corresponds to the partial ionization of hydrogen. A specially interesting case is displayed in Fig. 4 where the accretion rate is $\dot{M}=10^{-12}$ $M_{\odot} / \mathrm{yr}$, typical for an EBS disc in quiescence. There is a (multi-valued) optically thin solution with $T \lesssim 6600 \mathrm{~K}$ (zone $2 \mathrm{bis}$ in the figure). We have checked that this regime is also compatible with both $H / R \ll 1$ and $\mathcal{M}_{\mathrm{t}}<1$. We see that the hottest branch of this solution, which has already been investigated by Williams (1980), is thermally stable. 
Table 1. Quantity $\left(\partial \ln Q^{-} / \partial \ln T\right)_{\Sigma, \Omega}$ and inferred thermal stability for ideal opacity sources (disregarding self-gravitation). Values and comments in parenthesis concern the $\alpha$-prescription. Various cases are considered: $\tilde{\beta}=1(\tilde{\beta}$ is the ratio of the gas pressure to the total pressure); $\tilde{\beta}=\frac{1}{2}$ and $\tilde{\beta}=0$ (radiative pressure only); Thompson scattering only $\left(\kappa \equiv \sigma_{\mathrm{T}}\right)$; free-free opacity only $\left(\kappa \equiv \kappa_{\mathrm{ff}} \propto \rho T^{-7 / 2}\right)$; both absorption processes $\left(\right.$ with $x=\frac{\kappa_{\mathrm{ff}}}{\kappa_{\mathrm{ff}}+\sigma_{\mathrm{T}}} \leq 1$ )

\begin{tabular}{|c|c|c|c|c|c|c|c|}
\hline$\beta$ & opacity & \multicolumn{3}{|c|}{ optically thick limit (see Eq. (B.8)) } & \multicolumn{3}{|c|}{ optically thin limit (see Eq. (B.11)) } \\
\hline 1 & $\sigma_{\mathrm{T}}$ & -4 & stable & $(-3$, stable $)$ & -4 & stable & $(-3$, stable $)$ \\
\hline & & -8 & stable & $(-7$, stable $)$ & 0 & stable & $(+1$, unstable $)$ \\
\hline & $\sigma_{\mathrm{T}}+\kappa_{\mathrm{ff}}$ & $-4(1+x)<0$ & stable & & $-4(1-x) \leq 0$ & stable & \\
\hline$\frac{1}{2}$ & & $\begin{array}{r}-4 \\
55\end{array}$ & stable & $\left(-\frac{2}{3}\right.$, stable $)$ & -4 & stable & $\left(-\frac{2}{3}\right.$, stable $)$ \\
\hline & $\kappa_{\mathrm{ff}}$ & $\begin{array}{c}-\frac{55}{6} \\
-4-\frac{31}{1} x<0\end{array}$ & $\begin{array}{l}\text { stable } \\
\text { stable }\end{array}$ & $\left(-\frac{35}{6}\right.$, stable $)$ & $\begin{array}{c}-\frac{1}{8} \\
-4+\left(\frac{10-7 x^{2}}{0}+\frac{7}{9}\right) x<0\end{array}$ & stable & $\left(+\frac{5}{8}\right.$, \\
\hline 0 & & -4 & stable & $(+4$, unstable $)$ & -4 & stable & $(+4$, unstable $)$ \\
\hline & $\kappa_{\mathrm{ff}}$ & $-\frac{23}{2}$ & stable & $\left(-\frac{7}{2}\right.$, stable $)$ & $-\frac{1}{4}$ & stable & $\left(+\frac{1}{4}\right.$, unstable $)$ \\
\hline & $\sigma_{\mathrm{T}}+\kappa_{\mathrm{ff}}$ & $-4-\frac{15}{2} x<0$ & stable & & $-4+\left(\frac{8-1 x}{2+2 x}+\frac{7}{2}\right) x<0$ & stable & \\
\hline
\end{tabular}

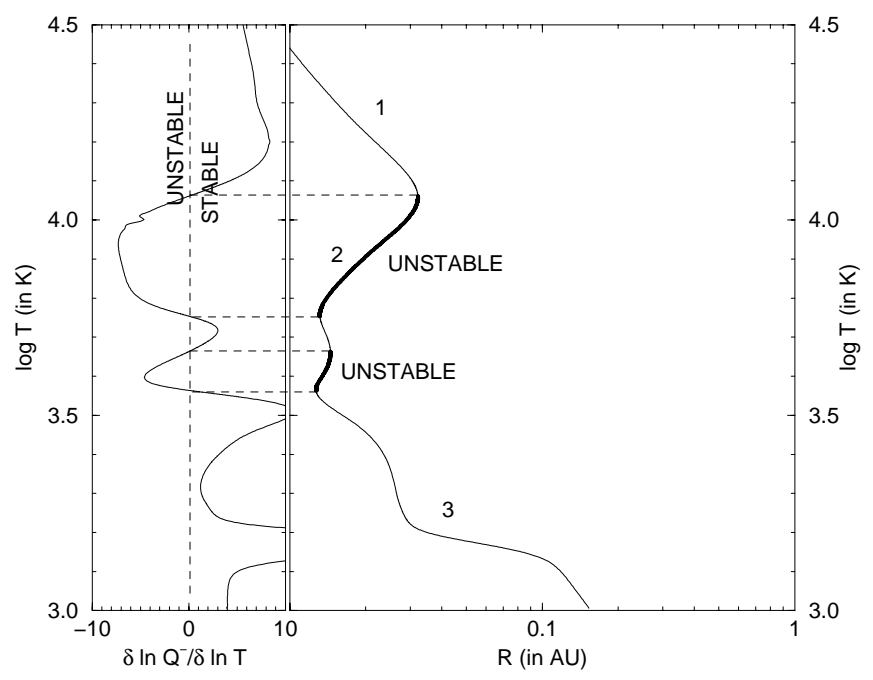

Fig. 3. Right panel: temperature radial profile (thin line) and thermally instable regions (bold line) in a "realistic" $\beta$-disc for $M=1 M_{\odot}$ and $\dot{M}=10^{-8} M_{\odot} /$ yr. Left panel: the quantity $\left(\partial \ln Q^{-} / \partial \ln T\right)_{\Sigma, \Omega}$ versus $T$ computed from Eq. (B.7)

Similar computations have been carried out for many input pairs $(M, \dot{M})$ corresponding to AGN, EBS and YSO discs and the overall conclusions are the following:

- the hot optically thick solution which extends down to the central object (zone 1 in Figs. 3 and 4 ) is always thermally stable (this includes the radiative pressure dominated solution);

- the region where hydrogen recombines (zone 2) is always unstable if the disc is optically thick;

- the hottest branch of the optically thin solution with $7000 \mathrm{~K} \gtrsim T \gtrsim 4000 \mathrm{~K}$ and $\mathrm{d} T / \mathrm{d} R<0$ (zone 2bis), when it exists, is always stable, even if hydrogen recombination occurs;

- the cold branches of the optically thin solution with $T \lesssim 4000 \mathrm{~K}$ and $\mathrm{d} T / \mathrm{d} R>0$ are always unstable;

- the outermost parts (zone 3) are globally stable. Thin unstable zones can be present.

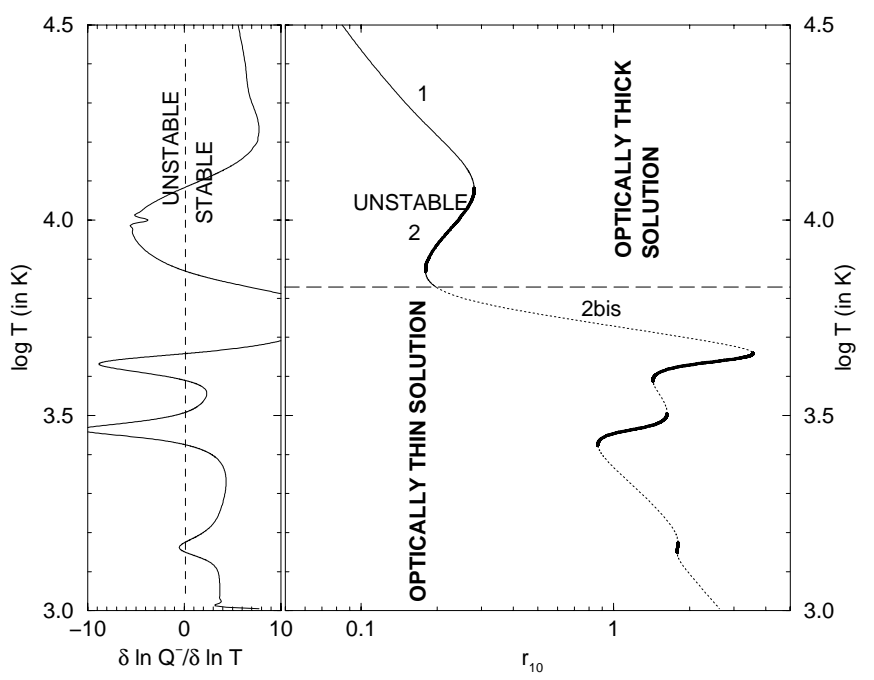

Fig. 4. Same legend as for Fig. 3 but for $\dot{M}=10^{-12} M_{\odot} / \mathrm{yr}$ typical of a quiescent EBS disc. The optically thick/thin limit is reached at $r_{10} \simeq 0.2$ where $T \simeq 6600 \mathrm{~K}$. The optical thickness $\tau$ goes through a minimum for $T \simeq 4500 \mathrm{~K}$ where $\tau \simeq 410^{-4}$

\section{Conclusion}

In this paper, we have examined some properties of thin accretion discs built with the $\beta$-prescription for the turbulent viscosity, which has been derived from the transport of angular momentum observed in differentially rotating laboratory flows (Richard \& Zahn 1999). This prescription may be applied whenever it predicts a turbulent velocity that is subsonic, and we have verified that, with the value we have taken for $\beta$ (i.e. $10^{-5}$ ), this condition is fulfiled in most discs, around stellar objects or massive black holes, except in the outer part of AGN discs where gas pressure dominates. Only radiatively cooled discs have been surveyed; it would be interesting to investigate ADAF-type solutions with this prescription. Let us recall that our conclusions have been obtained here with vertically averaged models, and it is plausible that a more realistic 2D-model would extend somewhat the domain of applicability of the $\beta$-prescription (Huré \& Richard 2000). 
Although the $\beta$-viscosity and the $\alpha$-viscosity may have comparable magnitudes, they have very different effects both on the structure and on the stability of steady Keplerian discs. An important property of $\beta$-discs is that they are viscously stable and do not tend to fragment into concentric rings, contrary to $\alpha$-discs. Also, unlike $\alpha$ discs, they are thermally stable for ideal cooling processes, such as Thomson scattering and free-free absorption, as was shown by Piran (1978). However, with more realistic opacities and equation of state, models of $\beta$-discs contain a thermally unstable region corresponding to the recombination of hydrogen, much like standard $\alpha$-discs. It remains to be seen with time-dependent models whether this instability can lead, as we suspect, to a limit cycle behavior.

Acknowledgements. The authors wish to thank the referee for his competent remarks and suggestions.

\section{Appendix A: Vertically averaged equations for a steady state Keplerian $\beta$-disc}

In a steady state Keplerian accretion disc where the viscosity is given by Eq. (2), the mass accretion rate $\dot{M}$ is linked to the surface density $\Sigma$ by the relation

$\dot{M}=3 \pi \beta \Omega R^{2} \Sigma$,

where $\Omega$ is the Keplerian angular velocity and $R$ the radius. The surface density is $\Sigma=2 \rho H$, with $\rho$ being the mass density and $H$ the total pressure scale height, which is determined from the equation of hydrostatic equilibrium

$\frac{P}{\rho H}=\Omega^{2} H(1+\zeta) \quad$ with $\quad \zeta=\frac{4 \pi G \rho}{\Omega^{2}}$,

where $\zeta$ represents the effect of self-gravity. The total pressure $P$ is the sum of the gas pressure $P_{\mathrm{g}}$ and of the radiation pressure $P_{\mathrm{r}}$ :

$P=P_{\mathrm{g}}+P_{\mathrm{r}}=\frac{P_{\mathrm{g}}}{\tilde{\beta}}$

We draw $P_{\mathrm{g}}$ from the perfect gas equation

$P_{\mathrm{g}}=\frac{\rho k T}{\mu \mathrm{m}_{\mathrm{H}}}$,

where $T$ is the midplane temperature and $\mu$ the mean mass per particle in units of the proton mass. The disc temperature in the equatorial plane is fixed by the balance $Q^{+}=Q^{-}$between viscous heating (Frank et al. 1992)

$Q^{+}=\frac{3 \Omega^{2} \dot{M}}{8 \pi}$

and radiative losses (Hubeny 1990)

$Q^{-}=\frac{8 \sigma T^{4}}{3 \kappa_{\mathrm{R}} \rho H+\frac{2}{\kappa_{\mathrm{P}} \rho H}+2 \sqrt{3}}$,

where $\kappa_{\mathrm{R}}$ and $\kappa_{\mathrm{P}}$ are respectively the Rosseland and Planck mean opacities (in $\mathrm{cm}^{2} \mathrm{~g}^{-1}$ ). The general expression for the radiative pressure is then

$P_{\mathrm{r}}=\frac{Q^{-}}{c}\left(\frac{1}{2} \kappa_{\mathrm{R}} \rho H+\frac{1}{\sqrt{3}}\right)$.
Note that in the optically thick limit the radiation pressure tends to its LTE value (labeled with an asterisk)

$P_{\mathrm{r}}=\frac{4 \sigma T^{4}}{3 c} \equiv P_{\mathrm{r}}^{*}$.

The above equation set can in fact be reduced to a system of two non linear algebraic equations with $\Sigma$ as the unknown. From Eqs. (A.1)-(A.7), the first equation is

$P^{*}-\pi G \Sigma^{2}-\frac{\pi^{6} \beta^{6} G^{4} M^{4}}{256 \dot{M}^{6}} \frac{\Sigma^{8}}{\rho}-\frac{16 \sigma T^{4}}{9 c \kappa_{\mathrm{R}} \kappa_{\mathrm{P}} \mathcal{S}(\Sigma)}=0$,

where the function $\mathcal{S}(\Sigma)$ is

$\mathcal{S}(\Sigma)=\frac{\Sigma^{2}}{2}+\frac{2 \Sigma}{\sqrt{3} \kappa_{\mathrm{R}}}+\frac{4}{3 \kappa_{\mathrm{R}} \kappa_{\mathrm{P}}}$.

The second equation is obtained from Eqs. (A.1), (A.5) and (A.6)

$\frac{\sigma \dot{M}^{5} T^{4}}{3^{14} \pi^{11} \kappa_{\mathrm{R}}}=G^{4} M^{4} \beta^{6} \mathcal{S}(\Sigma) \Sigma^{5}$.

We can follow the method described in Huré (1998) to solve the system of Eqs. (A.9) and (A.11). Note that the solution is valid in all cases: optically thick or thin, pressure dominated by gas or radiation, self-gravitating or not.

\section{A.1. The optically thick limit}

If we set $\mathcal{S}(\Sigma)=\frac{1}{2} \Sigma^{2}$ in Eqs. (A.9) and (A.11), and omit the last term of Eq. (A.9) we recover the optically thick limit $(\tau \gg 1)$, and there is a single equation to solve:

$\dot{M}^{12 / 7}+\lambda_{1} \dot{M}^{2 / 7}+\lambda_{0}=0$.

The coefficients $\lambda_{0}$ and $\lambda_{1}$ are respectively

$\lambda_{0}=16\left(\frac{\pi^{5 / 6} \beta \sigma M^{2 / 3}}{3 \sqrt{G} \kappa_{\mathrm{R}}}\right)^{6 / 7} \frac{T^{24 / 7}}{\rho}$ and

$\lambda_{1}=-\frac{3}{4}\left(\frac{3 \kappa_{\mathrm{R}} \pi \beta^{6} \sqrt{\pi G} M^{4}}{\sigma}\right)^{2 / 7} \frac{P^{*}}{T^{8 / 7}}$.

Note that the solution of Eq. (A.12) must satisfy $\tau \gg 1$.

\section{A.2. The optically thin limit}

In the optically thin limit $(\tau \ll 1), \mathcal{S}(\Sigma)=4 / 3 \kappa_{\mathrm{R}} \kappa_{\mathrm{P}}$ and the last term of Eq. (A.9) is identical to $P_{\mathrm{r}}^{*}$. There is also a single equation to solve, namely

$$
\begin{aligned}
{[4 \pi G \rho} & \left.+\left(\frac{16 \sigma \kappa_{\mathrm{P}}}{3 \beta G^{2 / 3} M^{2 / 3}} T^{4}\right)^{6 / 5}\right] \dot{M}^{2} \\
& -\frac{3 k \rho^{2}}{\mu \mathrm{m}_{\mathrm{H}} T^{3 / 5}}\left(\frac{6 \pi^{5} \beta^{6} G^{4} M^{4}}{\sigma \kappa_{\mathrm{P}}}\right)^{2 / 5}=0
\end{aligned}
$$

and its solution must be such that $\tau \ll 1$. 


\section{Appendix B: Criterion for the thermal instability}

Setting $\tau=\kappa_{\mathrm{R}} \Sigma+8 / 3 \kappa_{\mathrm{P}} \Sigma+4 / \sqrt{3}$, and differentiating Eq. (A.6) while keeping $\Sigma$ constant, we obtain

$$
\begin{aligned}
\left(\frac{\partial \ln Q^{-}}{\partial \ln T}\right)_{\Sigma, \Omega}= & 4-\frac{1}{\tau}\left[\tau_{\mathrm{R}} b_{\mathrm{R}}-\frac{8 b_{\mathrm{P}}}{3 \tau_{\mathrm{P}}}\right] \\
& -\frac{1}{\tau}\left[\tau_{\mathrm{R}} a_{\mathrm{R}}-\frac{8 a_{\mathrm{P}}}{3 \tau_{\mathrm{P}}}\right]\left(\frac{\partial \ln \rho}{\partial \ln T}\right)_{\Sigma, \Omega},
\end{aligned}
$$

where $\tau_{\mathrm{R}}=\kappa_{\mathrm{R}} \Sigma$ and $\tau_{\mathrm{P}}=\kappa_{\mathrm{P}} \Sigma$ are the total Rosseland and Planck optical thicknesses respectively, and

$$
\begin{aligned}
a_{\mathrm{R}}=\left(\frac{\partial \ln \kappa_{R}}{\partial \ln \rho}\right)_{T}, & b_{\mathrm{R}}=\left(\frac{\partial \ln \kappa_{R}}{\partial \ln T}\right)_{\rho}, \\
a_{\mathrm{P}}=\left(\frac{\partial \ln \kappa_{P}}{\partial \ln \rho}\right)_{T}, & b_{\mathrm{P}}=\left(\frac{\partial \ln \kappa_{P}}{\partial \ln T}\right)_{\rho} .
\end{aligned}
$$

Note that this expression does not depend on the actual viscosity prescription. For Thompson scattering, we have simply $a_{\mathrm{R}}=b_{\mathrm{R}}=a_{\mathrm{P}}=b_{\mathrm{P}}=0$, and for free-free opacity, $a_{\mathrm{R}}=a_{\mathrm{P}}=1$ and $b_{\mathrm{R}}=b_{\mathrm{P}}=-\frac{7}{2}$. For a combination of both sources, we have $a_{\mathrm{R}}=a_{\mathrm{P}}=x$ and $b_{\mathrm{R}}=b_{\mathrm{P}}=-\frac{7}{2} x$ where $x \equiv \kappa_{\mathrm{ff}} /\left(\kappa_{\mathrm{ff}}+\sigma_{\mathrm{T}}\right)$.

To compute the derivative $(\partial \ln \rho / \partial \ln T)_{\Sigma, \Omega}$, we use the two different expressions of the total pressure at the midplane. First, by differentiation of Eq. (A.2), keeping $\Sigma$ and $\Omega$ constant, we have

$$
\left(\frac{\partial \ln P}{\partial \ln T}\right)_{\Sigma, \Omega}=-\frac{1}{1+\zeta}\left(\frac{\partial \ln \rho}{\partial \ln T}\right)_{\Sigma, \Omega} .
$$

Note that $(\partial \ln \rho / \partial \ln T)_{\Omega, \Sigma}$ is always negative. Second, from Eq. (A.3) which is written

$$
P=P_{\mathrm{g}}+\frac{Q^{-}}{c}\left(\frac{\tau_{\mathrm{R}}}{4}+\frac{1}{\sqrt{3}}\right)
$$

and setting $\chi_{\rho}^{\mathrm{g}}=\left(\partial \ln P_{\mathrm{g}} / \partial \ln \mathrm{T}\right)_{\rho}, \chi_{\mathrm{T}}^{\mathrm{g}}=\left(\partial \ln P_{\mathrm{g}} / \partial \ln \rho\right)_{\mathrm{T}}$ and $\tilde{\beta}=P_{\mathrm{g}} / P$, we have

$$
\begin{aligned}
\left(\frac{\partial \ln P}{\partial \ln T}\right)_{\Sigma, \Omega}= & {\left[\tilde{\beta} \chi_{\mathrm{T}}^{\mathrm{g}}+(1-\tilde{\beta}) a_{\mathrm{R}} \frac{\tau_{\mathrm{R}}}{\tau_{\mathrm{R}}+\frac{4}{\sqrt{3}}}\right] \quad \text { (B.4) } } \\
& \times\left(\frac{\partial \ln \rho}{\partial \ln T}\right)_{\Sigma, \Omega}+\tilde{\beta} \chi_{\rho}^{\mathrm{g}}+(1-\tilde{\beta}) \\
& \times \frac{\tau_{\mathrm{R}}}{\tau_{\mathrm{R}}+\frac{4}{\sqrt{3}}} b_{\mathrm{R}}+(1-\tilde{\beta})\left(\frac{\partial \ln Q^{-}}{\partial \ln T}\right)_{\Sigma, \Omega} .
\end{aligned}
$$

Combining now Eqs. (B.2) and (B.4), we find

$$
\begin{aligned}
& -\left(\frac{\partial \ln \rho}{\partial \ln T}\right)_{\Sigma, \Omega}\left[\frac{1}{1+\zeta}+\tilde{\beta} \chi_{\mathrm{T}}^{\mathrm{g}}+(1-\tilde{\beta}) a_{\mathrm{R}} \frac{\tau_{\mathrm{R}}}{\tau_{\mathrm{R}}+\frac{4}{\sqrt{3}}}\right]= \\
& \tilde{\beta} \chi_{\rho}^{\mathrm{g}}+(1-\tilde{\beta}) \frac{\tau_{\mathrm{R}}}{\tau_{\mathrm{R}}+\frac{4}{\sqrt{3}}} b_{\mathrm{R}}+(1-\tilde{\beta})\left(\frac{\partial \ln Q^{-}}{\partial \ln T}\right)_{\Sigma, \Omega} .
\end{aligned}
$$

Finally, from Eqs. (B.1) and (B.5) and after rearrangement

$$
\begin{aligned}
\left(\frac{\partial \ln \rho}{\partial \ln T}\right)_{\Sigma, \Omega}= & {\left[4(1-\tilde{\beta})-(1-\tilde{\beta}) \frac{\tau_{\mathrm{R}} b_{\mathrm{R}}-\frac{8 b_{\mathrm{P}}}{3 \tau_{\mathrm{P}}}}{\tau}\right.} \\
& \left.+\tilde{\beta} \chi_{\rho}^{\mathrm{g}}+(1-\tilde{\beta}) \frac{\tau_{\mathrm{R}}}{\tau_{\mathrm{R}}+\frac{4}{\sqrt{3}}} b_{\mathrm{R}}\right] \\
& \times\left[(1-\tilde{\beta}) \frac{\tau_{\mathrm{R}} a_{\mathrm{R}}-\frac{8 a_{\mathrm{P}}}{3 \tau_{\mathrm{P}}}}{\tau}-\left(\frac{1}{1+\zeta}\right.\right. \\
& \left.\left.+\tilde{\beta} \chi_{\mathrm{T}}^{\mathrm{g}}+(1-\tilde{\beta}) a_{\mathrm{R}} \frac{\tau_{\mathrm{R}}}{\tau_{\mathrm{R}}+\frac{4}{\sqrt{3}}}\right)\right]^{-1}
\end{aligned}
$$

and so Eq. (B.1) becomes

$$
\begin{aligned}
\left(\frac{\partial \ln Q^{-}}{\partial \ln T}\right)_{\Sigma, \Omega}= & {\left[\tilde{\beta} \chi_{\rho}^{\mathrm{g}}+(1-\tilde{\beta}) \frac{\tau_{\mathrm{R}}}{\tau_{\mathrm{R}}+\frac{4}{\sqrt{3}}} b_{\mathrm{R}}\right.} \\
& +\left(\frac{1}{1+\zeta}+\tilde{\beta} \chi_{\mathrm{T}}^{\mathrm{g}}+(1-\tilde{\beta})\right. \\
& \left.\left.\times a_{\mathrm{R}} \frac{\tau_{\mathrm{R}}}{\tau_{\mathrm{R}}+\frac{4}{\sqrt{3}}}\right)\left(\frac{4 \tau-\tau_{\mathrm{R}} b_{\mathrm{R}}+\frac{8 b_{\mathrm{P}}}{3 \tau_{\mathrm{P}}}}{\tau_{\mathrm{R}} a_{\mathrm{R}}-\frac{8 a_{\mathrm{P}}}{3 \tau_{\mathrm{P}}}}\right)\right] \\
& \times\left[\frac { \tau } { \tau _ { \mathrm { R } } a _ { \mathrm { R } } - \frac { 8 a _ { \mathrm { P } } } { 3 \tau _ { \mathrm { P } } } } \left(\frac{1}{1+\zeta}+\tilde{\beta} \chi_{\mathrm{T}}^{\mathrm{g}}+\right.\right. \\
& \left.\left.\times(1-\tilde{\beta}) a_{\mathrm{R}} \frac{\tau_{\mathrm{R}}}{\tau_{\mathrm{R}}+\frac{4}{\sqrt{3}}}\right)+\tilde{\beta}-1\right]^{-1}
\end{aligned}
$$

It is then easy to derive from Eq. (B.7) the following asymptotic expressions:

- in the optically thick limit $\left(\tau \sim \tau_{\mathrm{R}}\right.$ and $\left.\tau_{\mathrm{P}} \gtrsim \tau_{\mathrm{R}} \gg 1\right)$

$$
-\left(\frac{\partial \ln Q^{-}}{\partial \ln T}\right)_{\Sigma, \Omega}=b_{\mathrm{R}}-4-a_{\mathrm{R}} \frac{\tilde{\beta} \chi_{\rho}^{\mathrm{g}}+4(1-\tilde{\beta})}{\tilde{\beta} \chi_{\mathrm{T}}^{\mathrm{g}}+\frac{1}{1+\zeta}}
$$

- in the radiative pressure dominated limit $(\tilde{\beta} \sim 0)$

$$
-\left(\frac{\partial \ln Q^{-}}{\partial \ln T}\right)_{\Sigma, \Omega}=b_{\mathrm{R}}-4-4 a_{\mathrm{R}}(1+\zeta) ;
$$

- in the gas pressure dominated limit $(\tilde{\beta} \sim 1)$

$$
-\left(\frac{\partial \ln Q^{-}}{\partial \ln T}\right)_{\Sigma, \Omega}=b_{\mathrm{R}}-4-a_{\mathrm{R}} \frac{1+\zeta}{2+\zeta}
$$

- in the optically thin limit $\left(\tau \sim \frac{8}{3 \tau_{\mathrm{P}}}\right.$ and $\left.\tau_{\mathrm{P}} \ll 1\right)$

$$
\begin{aligned}
& -\left(\frac{\partial \ln Q^{-}}{\partial \ln T}\right)_{\Sigma, \Omega}= \\
& -b_{\mathrm{P}}-4+a_{\mathrm{P}} \frac{\tilde{\beta} \chi_{\rho}^{\mathrm{g}}+4(1-\tilde{\beta})+(1-\tilde{\beta}) b_{\mathrm{P}}}{(1-\tilde{\beta}) a_{\mathrm{P}}+\tilde{\beta} \chi_{\mathrm{T}}^{\mathrm{g}}+\frac{1}{1+\zeta}} .
\end{aligned}
$$




\section{References}

Balbus, S. A., \& Hawley, J. F. 1991, ApJ, 376, 214

Biermann, P. L., \& Duschl, W. J. 1998, in Dynamics of Galaxies and Galactic Nuclei, Proc. Ser. vol. 2, Institut für Theoretische Astrophysik, 13

Cannizzo, J. K. 1993, in Accretion disks in compact stellar systems, ed. J. C. Wheeler (World Scientific), 6

Chandrasekhar, S. 1961, Proc. Nat. Acad. Sci., 46, 53

Duschl, W. J, Strittmatter, P. A., \& Biermann, P. L. 2000, A\&A, 357, 1123

Frank, J., King, A., \& Raine, D. 1992, in Accretion power in astrophysics, 2nd edition (Cambridge Univ. Press)

De Freitas Pacheco, J. A., \& Steiner, J. E. 1976, A\&SS, 39, 487

Gammie, C. F., \& Menou, K. 1998, ApJL, 492, 75

Hawley, J. F., \& Balbus, S. A. 1991, ApJ, 376, 223

Hubeny, I. 1990, ApJ, 351, 632

Huré, J. M., \& Richard, D. 2000, Proceedings of the EAS meeting held in Toulouse France, 1999, ed. H. Sol, \& B. RoccaVolmerange (EDP Sciences)
Huré, J. M. 1998, A\&A, 337, 625

Lasota, J.-P., \& Hameury, J.-P. 1998, in Accretion processes in astrophysical systems: some like it hot, Proceedings of the 8th AIP Conference, ed. S. T. Holt, \& T. R. Kallman, 351

Lin, D. N. C., \& Papaloizou, J. 1980, MNRAS, 191, 37

Lynden-Bell, D., \& Pringle, J. 1974, MNRAS, 168, 603

Piran, T. 1978, ApJ, 221, 652

Pringle, J. 1981, ARA\&A, 19, 137

Richard, D., \& Zahn, J.-P. 1999, A\&A, 347, 734

Shakura, N. I., \& Sunyaev, R. A. 1973, A\&A, 24, 337

Siemiginowska, A., Czerny, B., \& Kostyunin, V. 1996, ApJ, 458,491

Taylor, G. I. 1936, Proc. Roy. Soc. London A, 157, 546

Thompson, R. I., Strittmatter, P. A., Erickson, E. F., Witteborn, F. C., \& Strecker, D. W. 1977, ApJ, 218, 170

Tylenda, R. 1981, Acta Astron, 31, 127

Wendt, F. 1933, Ing. Arch., 4, 577

Williams, R. E. 1980, ApJ, 235, 939 